\title{
Melanomas of Unknown Primary May Have a Distinct Molecular Classification to Explain Differences in Patient Outcomes
}

\author{
Georgia M. Beasley, MD, MHSc \\ Duke University, Durham, NC
}

Although metastatic melanoma of unknown primary (MUP) is relatively uncommon, clinicians who routinely treat melanoma patients will encounter and manage patients with MUP, as approximately $3-8 \%$ of all metastatic melanoma patients will present with MUP. Historically, the management of patients with MUP has been similar to the management of patients with metastatic melanoma and a known primary (MKP). However, biologic observations differentiating MUP and MKP have been consistently reported, including better survival for patients with MUP than for MKP patients with the same corresponding tumor stage. ${ }^{1,2}$ Furthermore, patients with MUP may have a better response to immune checkpoint therapy than patients with MKP. ${ }^{3}$

The biologic basis for observations that MUP may have better outcomes than patients with MKP has largely consisted of hypotheses regarding immune-mediated control of the primary tumor in patients with MUP. However, indepth immunologic and tumor analysis is limited to support this hypothesis. In this issue of Annals of Surgical Oncology, De Andrade et al. ${ }^{4}$ report on the "Multidisciplinary Care for Melanoma of Unknown Primary: Experience in the Era of Molecular Profiling," which begins to investigate the unique molecular classification of tumors associated with clinical presentation of MUP.

Importantly, the mutations reported in this article on MUP patients include activating BRAF and TERT promoter mutations, suggesting that the MUPs in this study

(C) Society of Surgical Oncology 2020

First Received: 26 August 2020

Accepted: 27 August 2020;

Published Online: 10 September 2020

G. M. Beasley, MD, MHSc

e-mail: Georgia.beasley@duke.edu were consistent with a cutaneous origin. Similarly, in single-gene assays for BRAF mutations in 42 patients, the $52 \%$ rate for BRAF mutations $(n=22)$ appears similar to that for cutaneous MKP. However, despite a small sample, the rates for V600E (55\%) and V600K (27\%) seem somewhat discordant with those for MKP, with V600E making up a larger majority of activating mutations. Interestingly, V600K mutations appear to benefit less from BRAFi/MEKi therapy and often have a higher mutational load corresponding to an improved response to immunotherapy. ${ }^{5}$ Thus a higher rate for V600K mutations in MUP could explain the improved response to immunotherapy.

In addition to BRAF mutations, differences in TERT promoter mutations are seen in $46 \%$ of the patients with MUP $(n=11)$ in this study, whereas TERT promoter mutations have been reported for $85 \%$ (45/53) of patients with metastatic melanoma and 33\% (25/77) of patients with primary melanoma. ${ }^{6}$ Although it is important to consider that these differences may be the result of a small sample and selection bias, the observed differences in disease outcomes for MUP and MKP suggest possible underlying differences in the molecular composition of these two clinical entities that remain unknown. Molecular analysis could ultimately uncover key biologic determinants of tumor growth and proliferation unique to MUP.

Clinical observations frequently are the gateway to scientific discovery. The biology of MUP is observed to be distinct from that of MKP, with multiple reports indicating a better prognosis for MUP patients than for stage-matched MKP patients. Defining a distinct signature of MUP would ultimately result in better information, treatment, and management for patients presenting with MUP. The molecular profile of tumors in MUP may have key differences that result in different treatment strategies for MUP and MKP. In this article, De Andrade et al. ${ }^{4}$ have begun to 
explore these potential molecular differences between MUP and MKP. A larger cohort study analyzing the molecular profile of patients with MUP is warranted.

DISCLOSURE Georgia M. Beasley served on the advisory board of Regeneron in 2020.

\section{REFERENCES}

1. Bae JM, Choi YY, Kim DS, et al. Metastatic melanomas of unknown primary show better prognosis than those of known primary: a systematic review and meta-analysis of observational studies. J Am Acad Dermatol. 2015;72:59-70.

2. Lee CC, Faries MB, Wanek LA, Morton DL. Improved survival for stage IV melanoma from an unknown primary site. $J$ Clin Oncol. 2009;27:3489-95.

3. Gambichler T, Chatzipantazi M, Schröter U, Stockfleth E, Gedik C. Patients with melanoma of unknown primary show better outcome under immune checkpoint inhibitor therapy than patients with known primary: preliminary results. Oncoimmunology. 2019;8:e1677139.

4. De Andrade JP, Wong P, O'Leary MP, et al. Multidisciplinary care for melanoma of unknown primary: Experience in the era of molecular profiling. Ann Surg Oncol. 2020. https://doi.org/10.124 5/s10434-020-09112-2.

5. Pires da Silva I, Wang KYX, Wilmott JS, et al. Distinct molecular profiles and immunotherapy treatment outcomes of V600E and V600K BRAF-mutant melanoma. Clin Cancer Res. 2019;25:1272-9.

6. Horn S, Figl A, Rachakonda PS, et al. TERT promoter mutations in familial and sporadic melanoma. Science. 2013;339:959-61.

Publisher's Note Springer Nature remains neutral with regard to jurisdictional claims in published maps and institutional affiliations. 